\title{
Generalised Mycielski graphs and the Borsuk-Ulam theorem
}

\author{
Tobias Müller* \\ Johann Bernoulli Institute \\ Groningen University \\ The Netherlands \\ tobias.muller@rug.nl
}

\author{
Matěj Stehlík ${ }^{\dagger}$ \\ Laboratoire G-SCOP \\ Université Grenoble Alpes \\ France \\ matej.stehlik@grenoble-inp.fr
}

Submitted: Jan 18, 2019; Accepted: Sep 22, 2019; Published: Oct 11, 2019

(C) The authors. Released under the CC BY-ND license (International 4.0).

\begin{abstract}
Stiebitz determined the chromatic number of generalised Mycielski graphs using the topological method of Lovász, which invokes the Borsuk-Ulam theorem. Van Ngoc and Tuza used elementary combinatorial arguments to prove Stiebitz's theorem for 4-chromatic generalised Mycielski graphs, and asked if there is also an elementary combinatorial proof for higher chromatic number. We answer their question by showing that Stiebitz's theorem can be deduced from a version of Fan's combinatorial lemma. Our proof uses topological terminology, but is otherwise completely discrete and could be rewritten to avoid topology altogether. However, doing so would be somewhat artificial, because we also show that Stiebitz's theorem is equivalent to the Borsuk-Ulam theorem.
\end{abstract}

Mathematics Subject Classifications: 05C15, 55U10

\section{Introduction}

The Mycielski construction [10] is one of the earliest and arguably simplest constructions of triangle-free graphs of arbitrary chromatic number. Given a graph $G=(V, E)$, we let $M_{2}(G)$ be the graph with vertex set $V \times\{0,1\} \cup\{z\}$, where there is an edge $\{(u, 0),(v, 0)\}$ and $\{(u, 0),(v, 1)\}$ whenever $\{u, v\} \in E$, and an edge $\{(u, 1), z\}$ for all $u \in V$. It is an easy exercise to show that the chromatic number increases with each iteration of $M_{2}(\cdot)$.

*Partially supported by NWO grants 639.032.529 and 612.001.409. Part of this work was carried out while this author visited Laboratoire G-SCOP supported by ANR Project Stint (ANR-13-BS02-0007) and LabEx PERSYVAL-Lab (ANR-11-LABX-0025-01).

$\dagger$ Partially supported by ANR project Stint (ANR-13-BS02-0007), ANR project GATO (ANR-16CE40-0009-01), and by LabEx PERSYVAL-Lab (ANR-11-LABX-0025). Part of this work was carried out while this author visited Utrecht University, supported by NWO grant 639.032.529. 


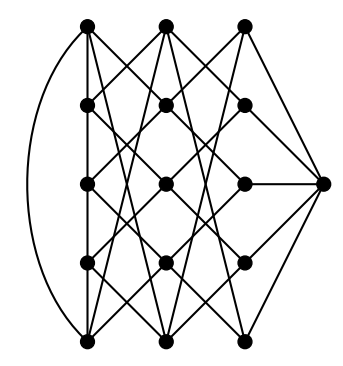

Figure 1: The graph $M_{3}\left(C_{5}\right) \cong M_{3}\left(M_{2}\left(K_{2}\right)\right) \in \mathcal{M}_{4}$.

The construction was generalised by Stiebitz [15] (see also [12, 5]), and independently by Van Ngoc [16] (see also [17]), in the following way. Given a graph $G=(V, E)$ and an integer $r \geqslant 1$, we define $M_{r}(G)$ as the graph with vertex set $V \times\{0, \ldots, r-1\} \cup\{z\}$, where there is an edge $\{(u, 0),(v, 0)\}$ and $\{(u, i),(v, i+1)\}$ whenever $\{u, v\} \in E$, and an edge $\{(u, r-1), z\}$ for all $u \in V$. The construction is illustrated in Figure 1.

If $r>2$, it is no longer true that the chromatic number increases with each iteration of $M_{r}(\cdot)$. For instance, it can be shown that if $\overline{C_{7}}$ is the complement of the 7 -cycle, then $\chi\left(M_{3}\left(\overline{C_{7}}\right)\right)=\chi\left(\overline{C_{7}}\right)=4$. However, Stiebitz [15] was able to show that the chromatic number does increase with each iteration of $M_{r}(\cdot)$ if we start with an odd cycle, or some other suitably chosen graph. For every integer $k \geqslant 2$, let us denote by $\mathcal{M}_{k}$ the set of all 'generalised Mycielski graphs' obtained from $K_{2}$ by $k-2$ iterations of $M_{r}(\cdot)$, where the value of $r$ can vary from iteration to iteration. Stiebitz [15] (see also [5, 8]) proved the following.

Theorem 1 (Stiebitz [15]). If $G \in \mathcal{M}_{k}$, then $\chi(G) \geqslant k$.

Stiebitz's proof is based on Lovász's [7] bound on the chromatic number in terms of the connectedness of the neighbourhood complex, which Lovász developed to prove Kneser's conjecture (see [8] for a comprehensive account). Lovász's bound uses the following result of Borsuk [2], usually known in the literature as the Borsuk-Ulam theorem.

Theorem 2 (Borsuk [2]). There exists no continuous antipodal mapping $f: S^{n} \rightarrow S^{n-1}$; that is, no continuous mapping such that $f(-x)=-f(x)$ for all $x \in S^{n}$.

To this day, no combinatorial proof of Theorem 1 is known (see [8, pp. 133]), except for the case $k=4$ [17]. At the end of their paper, Van Ngoc and Tuza [17] propose the following problem:

Finally, we would like to invite attention to the problem that no elementary combinatorial proof is known so far for the general form of Stiebitz's theorem, yielding graphs of arbitrarily large chromatic number and fairly large odd girth.

The answer to the problem depends on the interpretation of 'elementary combinatorial proof'. Does it mean a proof that is 'discrete' and does not rely on continuity? Or does it mean a 'graph theoretic' proof which avoids any topological concepts, such as triangulations of spheres? 
In this note we will give a new discrete proof of Theorem 1 based on a generalisation, due to Prescott and Su [11], of a classical lemma of Fan [4], and on a result of Kaiser and Stehlík [6]. Since the proofs of both these theorems are discrete, this provides a discrete proof of Theorem 1.

Triangulations of spheres are central to our proof, and rewriting the proof so as to avoid any topological concepts (as Matoušek [9] has done for the Lovász-Kneser theorem) is certainly possible, but seems somewhat artificial. Indeed, we show that Theorem 2 follows fairly easily from Theorem 1.

We would like to point out that our proof of Theorem 1 leads to a discrete proof of Schrijver's [13] sharpening of the Lovász-Kneser theorem [7], via the following result of Kaiser and Stehlík [6] (whose proof is entirely combinatorial). For a definition of $S G(n, k)$, we refer the reader to [6] or [8].

Theorem 3 (Kaiser and Stehlík [6]). For all integers $k \geqslant 1$ and $n>2 k$, there exists a graph $G \in \mathcal{M}_{n-2 k+2}$ homomorphic to $S G(n, k)$.

\section{Preliminaries}

Our graph theoretic terminology is standard and follows [1]. For an excellent introduction to topological methods in combinatorics, and all the topological terms used in this paper, see $[8]$.

Prescott and $\mathrm{Su}[11]$ introduced flags of hemispheres to prove a slight generalisation of Fan's combinatorial lemma [4]. A flag of hemispheres in $S^{n}$ is a sequence $H_{0} \subset \cdots \subset H_{n}$ where each $H_{d}$ is homeomorphic to a $d$-ball, $\left\{H_{0},-H_{0}\right\}$ are antipodal points, $H_{n} \cup-H_{n}=$ $S^{n}$, and for $1 \leqslant d \leqslant n$,

$$
\partial H_{d}=\partial\left(-H_{d}\right)=H_{d} \cap-H_{d}=H_{d-1} \cup-H_{d-1} \cong S^{d-1} .
$$

The polyhedron $|K|$ of a simplicial complex $K$ is defined as the union of all of its simplices. ${ }^{1}$ We say that $K$ is a triangulation of $|K|$ (or any space homeomorphic to it). A triangulation $K$ of $S^{n}$ is (centrally or antipodally) symmetric if $\sigma \in K$ whenever $-\sigma \in K$. A symmetric triangulation $K$ of $S^{n}$ is said to be aligned with hemispheres if we can find a flag of hemispheres such that for every $d$, there is a subcomplex of the $d$-skeleton of $K$ that triangulates $H_{d}$.

Given a simplicial complex $K$ and a labelling (map) $\lambda: V(K) \rightarrow \mathbb{Z} \backslash\{0\}$, we say a $d$-simplex $\sigma \in K$ is positive alternating if it has labels $\left\{+j_{0},-j_{1},+j_{2}, \ldots,(-1)^{d} j_{d}\right\}$, where $0<j_{0}<j_{1}<\cdots<j_{d}$. The following version of Fan's lemma [4] is a key ingredient of our proof.

Theorem 4 (Prescott and $\mathrm{Su}[11])$. Let $K$ be a symmetric triangulation of $S^{n}$ aligned with hemispheres, and let $\lambda: V(K) \rightarrow\{ \pm 1, \ldots, \pm k\}$ be a labelling such that $\lambda(-v)=-\lambda(v)$ for every vertex $v \in V(K)$, and $\lambda(u)+\lambda(v) \neq 0$ for every edge $\{u, v\} \in K$. Then there exists an odd number of positive alternating $n$-simplices. In particular, $k \geqslant n+1$.

\footnotetext{
${ }^{1}$ In the terminology of Matoušek [8], $K$ is a geometric simplicial complex.
} 
We remark that the proof in [11] is constructive and discrete, and that Fan's original result [4] imposes a more restrictive condition on the triangulation.

Suppose $K$ is a symmetric triangulation of $S^{n}$. A 2-colouring of $K$ is an assignment of two colours (black and white) to the vertices of $K$. The 2-colouring is said to be antisymmetric if antipodal vertices receive distinct colours, and it is proper if no $n$-simplex is monochromatic.

Given a symmetric triangulation $K$ of $S^{n}$ and a proper antisymmetric 2-colouring $\kappa$ of $K$, we denote by $\tilde{G}(K, \kappa)$ the graph obtained from the 1 -skeleton $K^{(1)}$ by deleting all monochromatic edges. If $\nu$ denotes the antipodal action on $\tilde{G}(K, \kappa)$, we set $G(K, \kappa)=$ $\tilde{G}(K, \kappa) / \nu$, and let $p: \tilde{G}(K, \kappa) \rightarrow G(K, \kappa)$ be the corresponding projection. Note that the graph $\tilde{G}(K, \kappa)$ is a bipartite double cover of $G(K, \kappa)$.

The first part of the following theorem is an immediate consequence of [6, Lemma 3.2 and Theorem 6.1], where the results are stated in terms of so-called quadrangulations of projective spaces. The second part is an easy exercice.

Theorem 5 (Kaiser and Stehlík [6]). Given $n \geqslant 1$, let $K$ be a symmetric triangulation of $S^{n}$ with a proper antisymmetric 2-colouring $\kappa$. For any $r \geqslant 1$, there exists a symmetric triangulation $K^{\prime}$ of $S^{n+1}$ with a proper antisymmetric 2 -colouring $\kappa^{\prime}$ such that $G\left(K^{\prime}, \kappa^{\prime}\right) \cong$ $M_{r}(G(K, \kappa))$. Moreover, if $K$ is aligned with hemispheres, then so is $K^{\prime}$.

\section{A combinatorial proof of Theorem 1}

Our proof of Theorem 1 is based on the following corollary of Theorem 4 .

Corollary 6. Let $K$ be a symmetric triangulation of $S^{n}$ aligned with hemispheres, and let $\lambda: V(K) \rightarrow\{ \pm 1, \ldots, \pm(n+1)\}$ be a labelling such that $\lambda(-v)=-\lambda(v)$ for every vertex $v \in V(K)$, and every $n$-simplex has vertices of both signs. Then there exists an edge $\{u, v\} \in K$ such that $\lambda(u)+\lambda(v)=0$.

Proof. Let $K, \lambda$ be as in the corollary and suppose, for the sake of contradiction, that $\lambda(u)+\lambda(v) \neq 0$ for every edge $\{u, v\} \in K$. We now define a new labelling $\mu: V(K) \rightarrow$ $\{ \pm 1, \ldots, \pm(n+1)\}$ by $\mu(v)=(-1)^{|\lambda(v)|} \lambda(v)$. Observe that

$$
\mu(-v)=(-1)^{|\lambda(-v)|} \lambda(-v)=-(-1)^{|\lambda(v)|} \lambda(v)=-\mu(v),
$$

and if $\mu(u)=-\mu(v)$, then $\lambda(u)=-\lambda(v)$, and therefore $\mu(u)+\mu(v) \neq 0$ for every edge $\{u, v\} \in K$. Hence $\mu$ satisfies the hypothesis of Theorem 4 . Therefore, there is an odd number of positive alternating $n$-simplices, i.e., simplices labelled $\left\{1,-2, \ldots,(-1)^{n} n\right.$, $\left.(-1)^{n+1}(n+1)\right\}$ by $\mu$. Hence, there is an odd number of simplices labelled $\{1,2, \ldots, n+1\}$ by $\lambda$. This contradicts the assumption that every $n$-simplex in $K$ has vertices of both signs. Hence, there exists an edge $\{u, v\} \in K$ such that $\lambda(u)+\lambda(v)=0$.

We are now ready to prove Theorem 1. 
Proof of Theorem 1. The case $k=2\left(G=K_{2}\right)$ and $k=3(G$ is an odd cycle) are trivial, so assume $k>3$ and let $G \in \mathcal{M}_{k}$. The graph $G$ is obtained from an odd cycle by $k-3$ iterations of $M_{r}(\cdot)$, where the value of $r$ can vary from iteration to iteration. By repeated applications of Theorem 5 ( $k-3$ applications to be exact), there exists a symmetric triangulation $K$ of $S^{k-2}$ aligned with hemispheres, and a proper antisymmetric 2-colouring $\kappa$ such that $G \cong G(K, \kappa)$. (To see this, observe that $M_{r}\left(K_{2}\right)$ is isomorphic to the odd cycle $C_{2 r+1}$, which is isomorphic to $G(K, \kappa)$, where $K$ is a symmetric triangulation of $S^{1}$-i.e., a graph-isomorphic to the cycle $C_{4 r+2}$, and $\kappa$ is a proper 2-colouring of $K$. By choosing any pair of antipodal vertices of $K$ to be the hemispheres $H_{0}$ and $-H_{0}$, it is clear that $K$ is aligned with hemispheres.) Let us say the colours used in $\kappa$ are black and white.

Consider any (not necessarily proper) $(k-1)$-colouring $c: V(G) \rightarrow\{1, \ldots, k-1\}$. By setting

$$
\lambda(v)= \begin{cases}+c(p(v)) & \text { if } v \text { is black } \\ -c(p(v)) & \text { if } v \text { is white }\end{cases}
$$

we obtain an antisymmetric labelling $\lambda: V(K) \rightarrow\{ \pm 1, \ldots, \pm(k-1)\}$ such that every $(k-2)$-simplex has vertices of both signs. By Corollary 6 , there exists an edge $\{u, v\} \in K$ such that $\lambda(u)+\lambda(v)=0$. Hence, the edge $\{p(u), p(v)\} \in E(G)$ satisfies $c(p(u))=|\lambda(u)|=$ $|\lambda(v)|=c(p(v))$, i.e., $c$ is not a proper colouring of $G$. This shows that $\chi(G) \geqslant k$.

\section{Equivalence of the theorems of Borsuk-Ulam and Stiebitz}

Let us recall the following construction due to Erdös and Hajnal [3]. The Borsuk graph $B G(n, \alpha)$ is defined as the (infinite) graph whose vertices are the points of $\mathbb{R}^{n+1}$ on $S^{n}$, and the edges connect points at Euclidean distance at least $\alpha$, where $0<\alpha<2$. Using Theorem 2, it can be shown that $\chi(B G(n, \alpha)) \geqslant n+2$ (in fact the two statements are equivalent, as noted by Lovász [7]). Furthermore, by using the standard $(n+2)$-colouring of $S^{n}$ based on the central projection of a regular $(n+1)$-simplex, it can be shown that $B G(n, \alpha)$ is $(n+2)$-chromatic for all $\alpha$ sufficiently large. In particular, Simonyi and Tardos [14] have shown that $B G(n, \alpha)$ is $(n+2)$-chromatic for all $\alpha \geqslant \alpha_{0}$, where $\alpha_{0}=2 \sqrt{1-1 /(n+3)}$.

We will need the following lemma.

Lemma 7. For every $n \geqslant 0$ and every $\delta>0$, there exists $G \in \mathcal{M}_{n+2}$ and a mapping $f: V(G) \rightarrow S^{n}$ such that $\|f(u)+f(v)\|<\delta$, for every edge $\{u, v\} \in E(G)$. In particular, $G \subset B G\left(n, \sqrt{4-\delta^{2}}\right)$.

Proof. The proof is by induction on $n$. We take $n=1$ as the base case, but we remark that the statement is also true for $n=0$, because $K_{2}$ is the only graph in $\mathcal{M}_{2}$, and the two vertices $u, v$ of $K_{2}$ can be placed at antipodal points of $S^{0}$, so $\|f(u)+f(v)\|=0$.

To see that the statement is true for $n=1$, observe that $\mathcal{M}_{3}$ is the family of odd cycles. The vertices of $C_{2 r+1}$ can be mapped to $S^{1}$ so that $f(u)$ and $-f(v)$ are at angular distance $\pi /(2 r+1)$, for every edge $\{u, v\} \in E(G)$. Therefore, $\|f(u)+f(v)\|=2 \sin (\pi /(4 r+2))$. 
As $r$ tends to infinity, $\|f(u)+f(v)\|$ tends to zero; in particular, for every $\delta>0$ there exists $r$ such that $\|f(u)+f(v)\|<\delta$ for every $\{u, v\} \in E\left(C_{2 r+1}\right)$.

Now suppose the theorem is true for $n \geqslant 1$. Fix a real number $\delta>0$. By the induction hypothesis, there exists $G \in \mathcal{M}_{n+2}$ and a mapping $f: V(G) \rightarrow S^{n}$ such that $\|f(u)+f(v)\|<\delta / 2$ for every $\{u, v\} \in E(G)$. We let $r \geqslant 2$ be a large integer, to be specified shortly in the proof, and we define a mapping $\bar{f}: V\left(M_{r}(G)\right) \rightarrow S^{n+1}$ by setting:

$$
\begin{aligned}
\bar{f}(z) & :=\left(0, \ldots, 0,(-1)^{r}\right), \\
\bar{f}((v, i)) & :=\left(f(v) \cos (\pi i / 2 r),(-1)^{i} \sin (\pi i / 2 r)\right) .
\end{aligned}
$$

Fix an arbitrary edge $\{x, y\} \in E\left(M_{r}(G)\right)$. We will show that $\|\bar{f}(x)+\bar{f}(y)\|<\delta$. First, if $x=(u, 0)$ and $y=(v, 0)$, for some $\{u, v\} \in E(G)$, then clearly $\bar{f}(x)=(f(u), 0)$ and $\bar{f}(y)=(f(v), 0)$, so $\|\bar{f}(x)+\bar{f}(y)\|=\|f(u)+f(v)\|<\delta / 2$.

Second, if $\{x, y\}=\{(u, i),(v, i+1)\}$, for some $\{u, v\} \in E(G)$, then applying the triangle inequality (twice) we get:

$$
\begin{aligned}
\|\bar{f}(x)+\bar{f}(y)\| \leqslant & \|f(u) \cos (\pi i / 2 r)+f(v) \cos (\pi(i+1) / 2 r)\| \\
& +|\sin (\pi i / 2 r)-\sin (\pi(i+1) / 2 r)| \\
\leqslant & \|f(u)+f(v)\| \cdot|\cos (\pi i / 2 r)| \\
& +\|f(v)\| \cdot|\cos (\pi(i+1) / 2 r)-\cos (\pi i / 2 r)| \\
& +|\sin (\pi i / 2 r)-\sin (\pi(i+1) / 2 r)| \\
\leqslant & \delta / 2+|\cos (t)-\cos (t+\varepsilon)|+|\sin (t)-\sin (t+\varepsilon)|,
\end{aligned}
$$

where $t=\pi i / 2 r, \varepsilon=\pi / 2 r$. Since sin and cos are uniformly continuous, having chosen $r$ sufficiently large, we can assume that $|\cos (t)-\cos (t+\varepsilon)|$, $|\sin (t)-\sin (t+\varepsilon)|<\delta / 4$ (for all $t \in \mathbb{R}$ in fact). So $\|f(x)+f(y)\|<\delta$ as required.

Finally, if $\{x, y\}=\{(u, r-1), z\}$, then we have

$$
\begin{aligned}
\|\bar{f}(x)+\bar{f}(y)\| & =\sqrt{\left.\cos ^{2}(\pi(r-1) / 2 r)+(1-\sin (\pi(r-1) / 2 r))\right)^{2}} \\
& <\delta
\end{aligned}
$$

where the inequality holds provided $r$ was chosen sufficiently large, using that $\cos (\pi(r-$ 1) $/ 2 r)$ approaches $\cos (\pi / 2)=0$ and $\sin (\pi(r-1) / 2 r))$ approaches $\sin (\pi / 2)=1$ as $r$ tends to infinity.

Thus, we have now shown that, provided $r$ was chosen sufficiently large, for every $\{x, y\} \in E\left(M_{r}(G)\right)$ we have $\|\bar{f}(x)+\bar{f}(y)\|<\delta$. The lemma follows by induction.

We will now show how Theorem 2 can be deduced from Theorem 1 and Lemma 7 .

Proof of Theorem 2. Suppose there exists a continuous antipodal map $f: S^{n} \rightarrow S^{n-1}$. Set $\varepsilon=1 / \sqrt{n+2}$. Since every continuous function on a compact set is uniformly continuous, there exists $\delta>0$ such that if $\|x-y\|<\delta$, then $\|f(x)-f(y)\|<2 \varepsilon$.

By Lemma 7, there exists $G \in \mathcal{M}_{n+2}$ and a mapping $g: V(G) \rightarrow S^{n}$ such that $\|g(u)+g(v)\|<\delta$, for every edge $\{u, v\} \in E(G)$. Therefore, the mapping $f \circ g: V(G) \rightarrow$ 
$S^{n-1}$ satisfies $\|f(g(u))+f(g(v))\|<2 \varepsilon$, for every edge $\{u, v\} \in E(G)$, so the Euclidean distance between $f(g(u))$ and $f(g(v))$ is

$$
\|f(g(u))-f(g(v))\|>2 \sqrt{1-\varepsilon^{2}}=2 \sqrt{1-1 /(n+2)} .
$$

Recalling that $\alpha_{0}=2 \sqrt{1-1 /(n+3)}$, we see that $G \subset B G\left(n-1, \alpha_{0}\right)$, and thus $\chi(G) \leqslant$ $\chi\left(B G\left(n-1, \alpha_{0}\right)\right)=n+1$. On the other hand, we have $\chi(G) \geqslant n+2$ by Theorem 1 . This contradiction proves that there is no continuous antipodal map $f: S^{n} \rightarrow S^{n-1}$.

\section{Acknowledgements}

We thank the referee for the comments which helped improve the quality of this paper.

\section{References}

[1] J. A. Bondy and U. S. R. Murty. Graph Theory, volume 244 of Graduate Texts in Mathematics. Springer, New York, 2008.

[2] K. Borsuk. Drei Sätze über die n-dimensionale euklidische Sphäre. Fund. Math., 20:177-190, 1933.

[3] P. Erdős and A. Hajnal. On chromatic graphs. Mat. Lapok, 18:1-4, 1967.

[4] K. Fan. A generalization of Tucker's combinatorial lemma with topological applications. Ann. of Math. (2), 56:431-437, 1952.

[5] A. Gyárfás, T. Jensen, and M. Stiebitz. On graphs with strongly independent colorclasses. J. Graph Theory, 46(1):1-14, 2004.

[6] T. Kaiser and M. Stehlík. Colouring quadrangulations of projective spaces. J. Combin. Theory Ser. B, 113:1-17, 2015.

[7] L. Lovász. Kneser's conjecture, chromatic number, and homotopy. J. Combin. Theory Ser. A, 25(3):319-324, 1978.

[8] J. Matoušek. Using the Borsuk-Ulam theorem. Universitext. Springer-Verlag, Berlin, 2003.

[9] J. Matoušek. A combinatorial proof of Kneser's conjecture. Combinatorica, 24(1):163-170, 2004.

[10] J. Mycielski. Sur le coloriage des graphes. Colloq. Math., 3:161-162, 1955.

[11] T. Prescott and F. E. Su. A constructive proof of Ky Fan's generalization of Tucker's lemma. J. Combin. Theory Ser. A, 111(2):257-265, 2005.

[12] H. Sachs and M. Stiebitz. On constructive methods in the theory of colour-critical graphs. Discrete Math., 74(1-2):201-226, 1989. Graph colouring and variations.

[13] A. Schrijver. Vertex-critical subgraphs of Kneser graphs. Nieuw Arch. Wisk. (3), 26(3):454-461, 1978.

[14] G. Simonyi and G. Tardos. Local chromatic number, Ky Fan's theorem and circular colorings. Combinatorica, 26(5):587-626, 2006. 
[15] M. Stiebitz. Beiträge zur Theorie der färbungskritischen Graphen. Habilitation thesis, Technische Hochschule Ilmenau, 1985.

[16] N. Van Ngoc. On graph colourings (Hungarian). PhD thesis, Hungarian Academy of Sciences, 1987.

[17] N. Van Ngoc and Zs. Tuza. 4-chromatic graphs with large odd girth. Discrete Math., 138(1-3):387-392, 1995. 14th British Combinatorial Conference (Keele, 1993). 